\title{
The Cycles of Alpha Centauri
}

\author{
Thomas R. Ayres ${ }^{1}$ \\ ${ }^{1}$ Center for Astrophysics \& Space Astronomy, University of Colorado, \\ 389-UCB (CASA), Boulder, Colorado, USA \\ email: Thomas. Ayres@Colorado.edu
}

\begin{abstract}
The main AB pair of the nearby Alpha Centauri triple system has one of the most extensive X-ray records of any cosmic object, stretching over three decades. The primary, $\alpha$ Cen A $(\mathrm{G} 2 \mathrm{~V})$, is a near twin of the Sun, with a similarly soft $(1-2 \mathrm{MK})$ corona. The secondary, $\alpha$ Cen $\mathrm{B}(\mathrm{K} 1 \mathrm{~V})$, is more active than the Sun, with a generally harder coronal spectrum. Here, spatially resolved measurements of the pair by Chandra's High Resolution Camera are compared, on a common basis, with previous pointings from ROSAT and XMM-Newton.
\end{abstract}

Keywords. X-rays: stars, stars: individual (HD 128620, HD 128621), stars: activity

\section{Introduction}

One of the remarkable properties of the Sun is the 11-year sunspot cycle, which in fact is a 22-year magnetic oscillation ("Hale Cycle") when polarity reversals of the mean field are taken into account. This signature property of solar magnetic activity is attributed to a global "dynamo" (e.g., Parker 1970). Because differential rotation acts as a catalyst, and because overturning turbulent gas flows are the ultimate power source of the dynamo, magnetic activity is crucially dependent on the stellar spin rate and whether the star is cool enough to support vigorous surface convection (see review by Güdel 2004).

The outward simplicity of the solar sunspot cycle is deceptive, however, and it has remained surprisingly resistant to theoretical modeling. The stubbornness of the solar example has encouraged the examination of analogous phenomena on other cool stars, to gain a broader perspective. The best known effort in this regard is the long term Ca II HK monitoring program begun by O.C. Wilson in the 1960's (Wilson 1978), to track cycles through subtle changes in chromospheric emission as the population of surface active regions waxes and wanes over long time scales. Most of the stellar cycles recovered to date are strikingly solar-like in HK amplitude and decadal duration (e.g., Radick et al. 1998). Unlike the activity itself, however, the stellar cycle periods do not seem to depend strongly on surface temperature or spin rate, except that the more active, fast rotators avoid cycles altogether, in favor of short-term stochastic chromospheric variability.

Surprisingly though, a debate still simmers over important observational properties of the solar cycle, especially its amplitude in soft X-rays associated with high energy coronal $(T \sim 1-10 \mathrm{MK})$ phenomena. The corona, in fact, is much more closely tied to the magnetic field than the Ca II chromosphere: the coronal gas is heated by a magnetic agency and bottled up in fine scale loop-like magnetic structures, while the chromosphere is heated partly by acoustic shocks. Consequently, the solar HK index varies only about $25 \%$ over the solar cycle, while coronal X-rays can change by factors of several to a hundred, depending on the energy band, although the details remain in some dispute. For example, Judge, Solomon, \& Ayres (2003) deduced a modest factor of $\sim 6$ change in the solar 0.1-2.4 keV X-ray luminosity over the rise of current Cycle 23, based on 
ultra-soft X-ray photometry from the SNOE satellite; compared with the more extreme $\sim 30 \times$ variations inferred from higher energy experiments like Yohkoh SXT (Acton 1996).

The X-ray cycle amplitude is a pivotal aspect of solar activity, because coronal ionizing radiations strongly influence the heliosphere in general, and Earth's upper atmosphere ("ionosphere") in particular. Indeed, the whole question of the evolution of the solar ionizing flux (and coronal wind) is central to the development of planetary atmospheres in the early solar system (e.g., Ayres 1997, and references to previous work therein), as well as young exoplanets around other stars; main topics of this Symposium.

Fundamentally, our few decades view of the Sun's X-cycle represents just a glimpse of what that variability might be over the longer term, in the past and into the future. Again, one is tempted to turn to the stars for insight. Discouragingly little is known about stellar X-cycles, however. The best examples, in terms of sample sizes, typically are two or three well separated surveys of young open clusters like the Hyades, where the inter-epoch variations appear to be dominated by flares (e.g., Stern 2001).

There are, however, a few cases of dedicated coronal monitoring programs on solarage field stars including the G subgiant HD 81809 (Favata et al. 2004), 61 Cyg AB (K5 V+K7 V; Hempelmann et al. 2006), and most notably the close-by $\alpha$ Centauri triple system (G2 V [A], K1 V [B], M5 V [C, also known as Proxima]). At a distance of just over a parsec, the main $\mathrm{AB}$ pair of $\alpha$ Cen has received considerable attention from four generations of X-ray observatories, beginning with its discovery (albeit unresolved) by HEAO-1 (Nugent \& Garmire 1978); separation into distinct coronal sources by Einstein (Golub et al. 1982); several campaigns with ROSAT (Schmitt \& Liefke 2004); and continuing into the contemporary eras of XMM-Newton (Robrade, Schmitt, \& Favata 2005) and Chandra (Ayres et al. 2008; Ayres 2009). The Robrade et al. study reported a sharp decline in the X-ray count rate of the primary in early 2005-a "fainting" of its corona, as the authors put it - which motivated the subsequent Chandra work. The $\alpha$ Cen AB orbit has been closing rapidly over the past decade, and in 2005 was only barely resolvable by $X M M-N e w t o n$, although even at minimum separation (4" , circa 2016) still will be easy for the sharper $1^{\prime \prime}$ gaze of Chandra. The latter campaign included direct imaging of the pair at roughly six month intervals from 2005 October to the present (most recent observation: 2009 May; one more scheduled for 2009, and two additional pointings in 2010). The Chandra campaign also included a pivotal deep transmission grating spectrum in mid-2007. It showed clearly that the steep decline of $\alpha$ Cen A seen by $X M M-N e w t o n$ mainly was the result of a loss of the highest temperature components of the primary's corona rather than a truly complete collapse of its hot outer atmosphere.

The present report reviews the progress made to date since the Ayres (2009) study.

\section{Observations}

Figure 1 illustrates time-lapse Chandra imaging of $\alpha$ Centauri over the period 20002010. $\alpha$ Cen A (G2 V) is to the upper left in each image pair, while its optically fainter companion (B: K1 V) is to the lower right. The peak intensity of $\alpha$ Cen B is about 2 counts $\mathrm{s}^{-1}$, a relatively bright Chandra source. Thin squiggly curves are combined orbital, parallactic, and proper motion tracks. Conspicuous 'wobbles' in the observed $\mathrm{X}$-ray spots represent the $0.8^{\prime \prime}$ annual parallax, easily captured by the high astrometric precision of Chandra. Note the rapid closing of the orbital separation in recent years. In the gap between the first Chandra pointing in 1999.98, and the beginning of the new HRC-I series in 2005.81, the XMM-Newton monitoring program, mentioned earlier, reported the surprising, steep decline in A's coronal X-rays. 


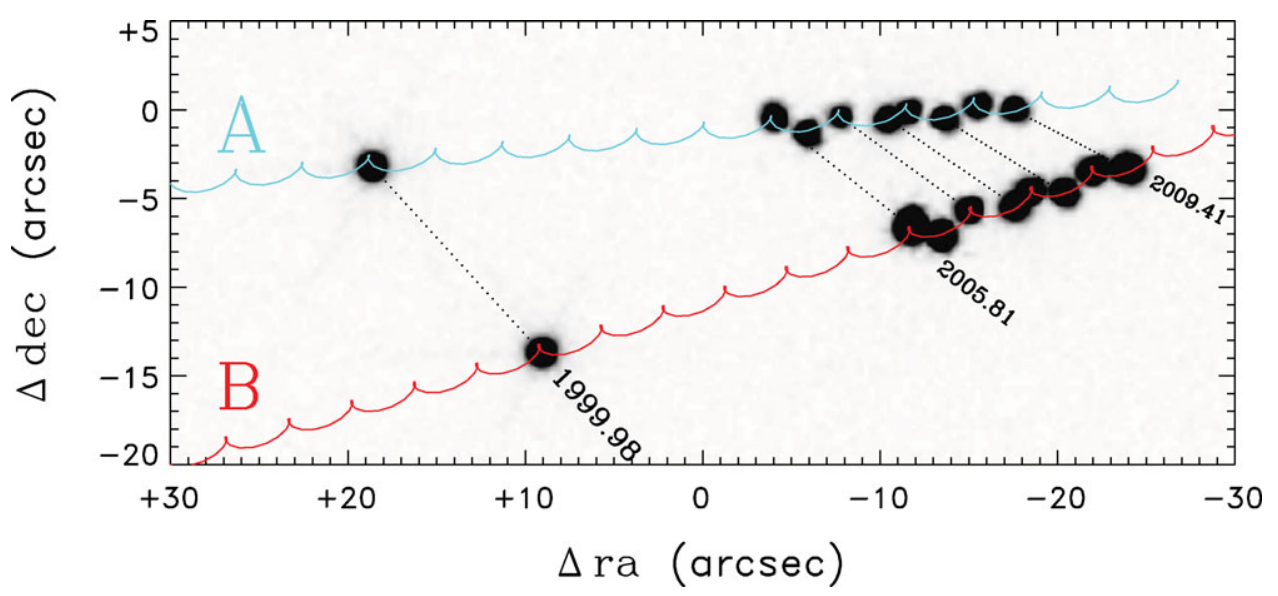

Figure 1. Chandra HRC imaging of $\alpha$ Centauri.

Figure 2 is the full X-ray time series of $\alpha$ Cen from ROSAT (1995-98 [upper panel: 1996]), and more recently XMM-Newton and Chandra (lower panel: the two dense ROSAT campaigns now are represented by time averages) for the reference energy band $0.2-2 \mathrm{keV}$. Blue points (lower) are for $\alpha$ Cen A, red points (upper) for B. Note the occasional "flares" in the B record during the two dense ROSAT sequences in 1996. The jagged hatched tracks from 2003-2007 are for XMM-Newton (semiannual measurements). Energy conversion factors were based on specialized DEM models derived for a range of possible activity states of the two stars. Furthermore, the soft response of the $X M M-N e w t o n$ EPIC cameras was progressively suppressed until a reasonable agreement was achieved with the overlapping Chandra points. Since EPIC must use the thick optical blocking filter for stars as bright as the $\alpha$ Cen pair (to control CCD "red leak"), it is plausible that the true soft response might be overestimated.

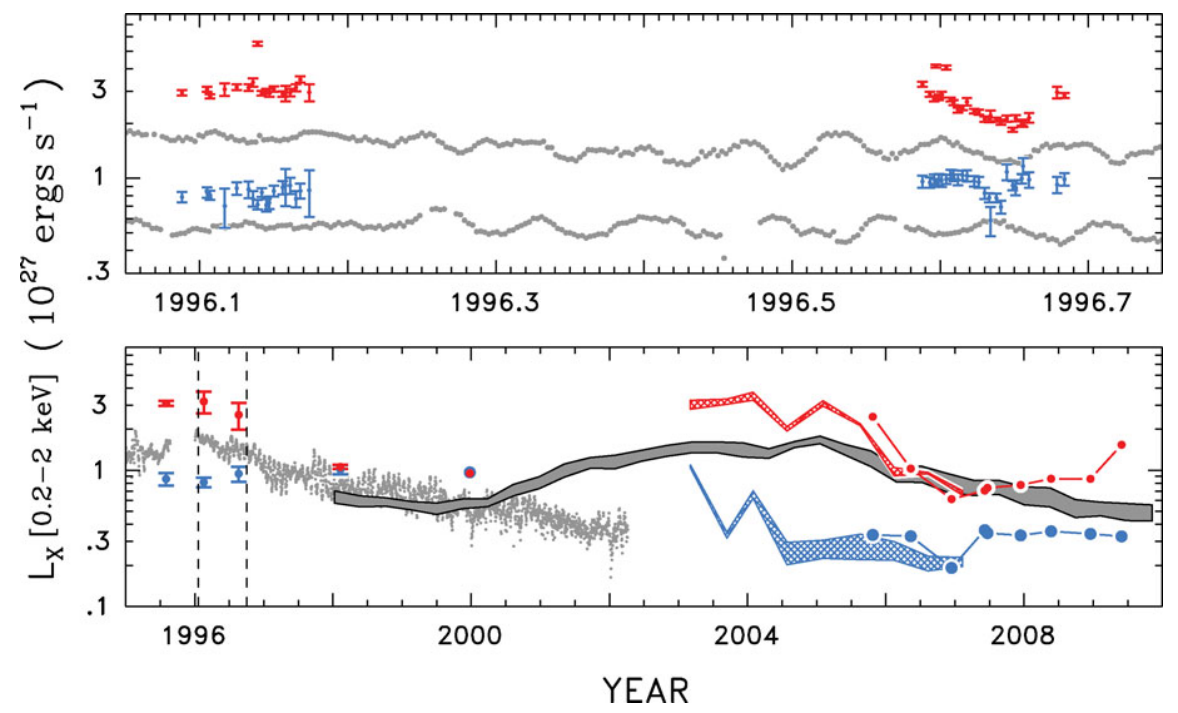

Figure 2. Long term history of $\alpha$ Cen X-rays. 
Gray points and the smooth shaded trace depict the estimated behavior of solar Xrays (during previous Cycle 23) in this energy band, shifted in time to roughly match various features of the $\alpha$ Cen series. In the top panel, the upper solar trace is for cycle maximum, the lower for minimum: rotational modulations are clearly visible at both extremes. Although $X M M-N e w t o n$ witnessed what appeared to be a dramatic 'fainting' of $\alpha$ Cen A in late 2004, the contemporary Chandra measurements, interpreted in light of the LETGS spectra of the AB pair, show that the drop of the primary from its historical high in 2000 has been relatively modest, smaller in fact than typical solar cycle changes.

\section{Conclusions}

The combined time series suggests that $\alpha$ Cen B was near X-ray maximum in the mid1990 's, minimum in the late-1990's, peaked again in 2004-2005, then was declining in 2006-2007, bottomed out in 2008, and by mid-2009 appears to be rising again. B's roughly 8-year X-ray modulation is shorter than the solar 11-year sunspot period, although the cycle depth is similar (factor of 5 in $0.2-2 \mathrm{keV}$ band). Meanwhile, $\alpha$ Cen A showed minimal variability during 1995-2000, a decline in 2003-2005, and presently is mired in an activity lull, somewhat down from the mid-1990's level. $\alpha$ Cen A is behaving like the Maunder minimum Sun, a "flat activity" star if you will (Judge \& Saar 2007).

Aside from the details of the twin X-cycles, the "smoking gun" Chandra transmission grating spectrum in mid-2007 emphasized that much of the coronal radiative output of cool-corona objects like the Sun falls at longer wavelengths than are recorded efficiently by contemporary stellar instruments, rendering $L_{\mathrm{X}}$ and cycle depth strongly dependent on the exact energy span of the measurements. This has broad-reaching impact on any discussions of coronal heating requirements, and the changes over starspot cycles.

\section{Acknowledgements}

This work was supported by the Chandra Guest Investigator program.

\section{References}

Acton, L. 1996, in: R. Pallavicini \& A. K. Dupree (eds.), Ninth Cambridge Workshop on Cool Stars, Stellar Systems, and the Sun, ASP Conf. Proc. 109, (San Francisco: ASP), p. 45

Ayres, T. R. 1997, JGR, 102, 1641

Ayres, T. R., Judge, P. G., Saar, S. H., \& Schmitt, J. H. M. M. 2008, ApJ, 678, L121

Ayres, T. R. 2009, ApJ, 696, 1931

Favata, F., et al. 2004, A\&BA, 418, L13

Golub, L., Harnden, F. R., Jr., Pallavicini, R., Rosner, R., \& Vaiana, G. S. 1982, ApJ, 253, 242

Güdel, M. 2004, A\&SAR, 12, 71

Hempelmann, et al. 2006, A\& A, 460, 261

Judge, P. G. \& Saar, S. H. 2007, ApJ, 663, 643

Judge, P. G., Solomon, S. C., \& Ayres, T. R. 2003, ApJ, 593, 534

Nugent, J. \& Garmire, G. 1978, ApJ, 226, L83

Parker, E. N. 1970, ApJ, 162, 665

Radick, R. R., Lockwood, G. W., Skiff, B. A., \& Baliunas, S. L. 1998, ApJS, 118, 239

Robrade, J., Schmitt, J. H. M. M., \& Favata, F. 2005, A\&A, 442, 315

Schmitt, J. H. M. M. \& Liefke, C. 2004, A\& A, 417, 651

Stern, R. A. 2001, Advances in Space Research, 26, 1723

Wilson, O. C. 1978, ApJ, 226, 379 